\title{
Riemannian Metric and Geometric Mean for Positive Semidefinite Matrices of Fixed Rank
}

\author{
Silvère Bonnabel* $\quad$ Rodolphe Sepulchre ${ }^{\dagger}$
}

\begin{abstract}
This paper introduces a new metric and mean on the set of positive semidefinite matrices of fixed-rank. The proposed metric is derived from a well-chosen Riemannian quotient geometry that generalizes the reductive geometry of the positive cone and the associated natural metric. The resulting Riemannian space has strong geometrical properties: it is geodesically complete, and the metric is invariant with respect to all transformations that preserve angles (orthogonal transformations, scalings, and pseudoinversion). A meaningful approximation of the associated Riemannian distance is proposed, that can be efficiently numerically computed via a simple algorithm based on SVD. The induced mean preserves the rank, possesses the most desirable characteristics of a geometric mean, and is easy to compute.
\end{abstract}

\section{Introduction}

Positive definite matrices have become fundamental computational objects in many areas of engineering and applied mathematics. They appear as covariance matrices in statistics, as elements of the search space in convex and semidefinite programming, as kernels in machine learning, and as diffusion tensors in medical imaging, to cite a few. Computing with positive definite matrices involves approximations, interpolation, filtering, and estimation, leading to a realm of metric-based algorithms. In the recent years, it has been increasingly recognized that the Euclidean distance does not suit best the set of positive definite matrices - the positive symmetric cone $\mathrm{P}_{\mathrm{n}}$ - and that working with the proper geometry does matter in computational problems.

${ }^{*}$ Department of Electrical Engineering and Computer Science, University of Liège, B4000 Liège, Belgium (bonnabel@montefiore.ulg.ac.be, r.sepulchre@ulg.ac.be). 
The (non-Euclidean) natural metric of the positive cone [13] proceeds from the rich quotient geometry of this set and its structure of reductive homogeneous space (see Appendix 7.2 for more details). The resulting "natural" metric is invariant to the action (by congruence) of the general linear group, a feature at the core of many desirable properties. The associated Riemannian mean can be called geometric rather than arithmetic. Recent contributions that have advocated the use of this metric in applications include $[26,14,34]$ for tensor computing in medical imaging and [7] for radar processing. More theoretical results can be found in [21, 4, 6, 28]. Most notably, the natural metric corresponds to the Fisher information metric for the multivariate normal distribution [10,31]. The comprehensive paper [32] uses this result to derive an intrinsic Cramér-Rao bound for the Gaussian covariance matrix estimation problem. Finally, the natural metric coincides with the metric defined by the natural self-concordant logarithmically homogeneous barrier $(-\log \operatorname{det} A)$ on the symmetric cone (which is a convex set) in optimization $[9,22]$. In particular, the interest in short-step methods relies on the property that $\mathrm{P}_{\mathrm{n}}$ with its natural metric is geodesically complete, i.e., every maximal geodesic is defined for all $t \in \mathbb{R}$, and thus the boundary can not be reached in finite time.

Because matrix algorithms tend to be applied to computational problems of ever-increasing size, they need to be adapted to remain tractable. Typical matrix computations (like SVD, EVD, QR factorization, etc.) require $O\left(n^{3}\right)$ operations for a positive definite matrix of size $n$, which limits their use in large-scale problems. A sensible remedy is to work with low-rank approximations instead. A rank $p$ approximation of a positive definite matrix can be factored as $A=Z Z^{T}$, where the matrix $Z \in \mathbb{R}^{n \times p}$ is of much reduced size if $p \ll n$, leading to a reduction of the numerical cost of typical matrix operations from $O\left(n^{3}\right)$ to $O\left(n p^{2}\right)$. If $p$ is kept to a moderate value, the complexity of the resulting algorithms grows only linearly with the size of the problem.

The natural metric developed for positive definite matrices is only valid for full-rank matrices. The goal of this paper is to extend the natural metric of the positive cone to the set $\mathrm{S}^{+}(\mathrm{p}, \mathrm{n})$ of symmetric positive semidefinite matrices of fixed-rank $p<n$. The set $\mathrm{S}^{+}(\mathrm{p}, \mathrm{n})$ admits a quotient geometry that generalizes the quotient geometry of the cone in a way that preserves most of the desirable properties of the cone geometry. Motivated by the natural metric of the positive cone, the proposed geometry differs from the quotient geometry recently proposed [3]. The resulting "natural" metric - which, to the best of the authors' knowledge, has not appeared in the literature previously — preserves not all but a remarkably large number of invariance properties of the natural metric in the cone. More precisely, it is invariant under all transformations that preserve angles, that is, rota- 
tions, scalings, and pseudo-inversion. Endowed with this metric $\mathrm{S}^{+}(\mathrm{p}, \mathrm{n})$ is geodesically complete. Moreover, we propose a meaningful and numerically efficient approximation of the Riemannian distance. The induced notion of mean is is shown to present all the desirable properties of a geometric mean.

The proposed natural metric on $\mathrm{S}^{+}(\mathrm{p}, \mathrm{n})$ is viewed as an important step to generalize several existing algorithms for positive definite matrices to the semidefinite case. This applies not only to the computational problems mentioned previously, but also to a growing realm of matrix nearness problems based on the use of Bregman divergences. We mention in particular the recent paper [11] that leaves as an open question the characterization of different types of projections to compute distances onto important sets of matrices, such as the positive semidefinite cone.

The rest of the paper is organized as follows. In section 2 we summarize the existing work on the positive definite cone $\mathrm{P}_{\mathrm{n}}$, concentrating on the features most relevant for the paper. To gain insight on the main issues faced when extending the geometry of $\mathrm{P}_{\mathrm{n}}$ to $\mathrm{S}^{+}(\mathrm{p}, \mathrm{n})$, section 3 focuses on the simplest case of $2 \times 2$ matrices of rank 1 . A metric extending the natural metric on the cone is derived using polar decomposition. We provide geometrical and physical justifications for the associated invariance, distance, and mean properties.

Building upon the polar decomposition of vectors in the plane, we develop in section 4 a quotient geometry for $\mathrm{S}^{+}(\mathrm{p}, \mathrm{n})$. The resulting natural metric decomposes as the sum of the natural metric on the cone and the standard metric of the Grassman manifold. We prove that $\mathrm{S}^{+}(\mathrm{p}, \mathrm{n})$ endowed with this metric is a Riemannian manifold.

Section 5 is devoted to the Riemannian distance associated to the metric. Because the explicit calculation of geodesics is out of reach, we construct special curves that approximate the geodesics. The length of those curves can be calculated by a SVD based algorithm of numerical complexity $O\left(n p^{2}\right)$. It provides a meaningful notion of closeness in $\mathrm{S}^{+}(\mathrm{p}, \mathrm{n})$ which inherits the invariance properties of the Riemannian distance. The singularities of this measure are also characterized. As an aside, the manifold $S^{+}(p, n)$ is proved to be geodesically complete.

Section 6 provides a new definition of geometric mean between two matrices of $\mathrm{S}^{+}(\mathrm{p}, \mathrm{n})$ based on the measure of closeness. We prove that the mean preserves the rank, we argue it deserves the appellation "geometric", and we prove it is a generalization to rank-deficient positive symmetric matrices of the mean associated with the natural metric on the positive cone. From a computational viewpoint, a main advantage of the metric is that, after a suitable SVD, the computations of the closeness and mean decouple into two separate problems: computing a Riemannian distance and mean 1 . in the 
(lower dimension) cone $\mathrm{P}_{\mathrm{p}}$ and 2. in the Grassman manifold of subspaces of dimension $p$ in $\mathbb{R}^{n}$.

Concluding remarks and perspectives are discussed in section 7 .

\section{$1.1 \quad$ Notation}

- $\mathrm{P}_{\mathrm{n}}$ is the set of symmetric positive definite $n \times n$ matrices.

- $\mathrm{S}^{+}(\mathrm{p}, \mathrm{n})$ is the set of symmetric positive semidefinite $n \times n$ matrices of rank $p \leq n$. We will only use this notation in the case $p<n$.

- $\mathrm{Gl}(\mathrm{n})$ is the general linear group, that is, the set of invertible $n \times n$ matrices.

- $\mathbb{R}_{*}^{n \times p}$ is the set of full rank $n \times p$ matrices.

- $\mathrm{V}_{\mathrm{n}, \mathrm{p}}=\mathrm{O}(\mathrm{n}) / \mathrm{O}(\mathrm{n}-\mathrm{p})$ is the Stiefel manifold; i.e., the set of $n \times p$ matrices with orthonormal columns: $U^{T} U=I_{p}$.

- $\operatorname{Gr}(\mathrm{p}, \mathrm{n})$ is the Grassman manifold, that is, the set of $p$-dimensional subspaces of $\mathbb{R}^{n}$. It can be represented by the equivalence classes $\mathrm{V}_{\mathrm{n}, \mathrm{p}} / \mathrm{O}(\mathrm{p})$.

- $\operatorname{Sym}(\mathrm{n})$ is the vector space of symmetric $n \times n$ matrices.

- $\operatorname{diag}\left(\lambda_{1}, \ldots, \lambda_{n}\right)$ is the $n \times n$ matrix with the $\lambda_{i}$ 's on its diagonal. $I=$ $\operatorname{diag}(1, \ldots, 1)$ is the identity matrix.

- $\operatorname{range}(A)$ is the subspace of $\mathbb{R}^{n}$ spanned by the columns of $A \in \mathbb{R}^{n \times n}$.

- $T_{X} \mathcal{M}$ is the tangent space to the manifold $\mathcal{M}$ at $X$.

\section{Riemannian distances and geometric means on the symmetric cone}

The geometry of the $n$-dimensional symmetric cone $\mathrm{P}_{\mathrm{n}}$ has been well-studied in the literature. This section reviews some of its relevant features in view of the main developments of the present paper. Given a matrix $A \in \mathrm{P}_{\mathrm{n}}$, a starting point is the matrix factorization

$$
A=Z Z^{T}=(U R)(U R)^{T}=U R^{2} U^{T}=R^{\prime 2},
$$


where $A \in \mathrm{P}_{\mathrm{n}}, Z \in \mathrm{Gl}(\mathrm{n}), R, R^{\prime} \in \mathrm{P}_{\mathrm{n}}, U \in \mathrm{O}(\mathrm{n})$. The right and left polar decompositions $Z=U R=R^{\prime} U$ are unique [15], but the factorization $A=$ $Z Z^{T}$ is unaffected by orthogonal transformations $Z \mapsto Z O$ with $O \in \mathrm{O}(\mathrm{n})$. The matrix equalities (1) underline the quotient geometry of the cone $\mathrm{P}_{\mathrm{n}}$.

$$
P_{n} \cong \mathrm{Gl}(\mathrm{n}) / \mathrm{O}(\mathrm{n}) \cong\left(\mathrm{O}(\mathrm{n}) \times \mathrm{P}_{\mathrm{n}}\right) / \mathrm{O}(\mathrm{n}) .
$$

The characterization (2) encodes the rich geometry of $\mathrm{P}_{\mathrm{n}}$ as a reductive homogeneous space, as discussed in $[17,32]$. The most relevant consequence of this feature to the present context is the existence of a $\mathrm{Gl}(\mathrm{n})$-invariant metric on the manifold $\mathrm{Gl}(\mathrm{n}) / \mathrm{O}(\mathrm{n})$. This metric is called the natural metric on the symmetric cone [13]. Up to a scaling factor it is also known as the affine-invariant metric [26], the Siegel metric in symplectic geometry, and it coincides with the metric given by the Fisher information matrix for Gaussian covariance matrix estimation [32]. We briefly summarize how it is derived and its main properties in the present context.

In view of (1), $\mathrm{Gl}(\mathrm{n})$ has a transitive action on $\mathrm{P}_{\mathrm{n}}$ via congruence

$$
A \mapsto L A L^{T},
$$

where $L \in \mathrm{Gl}(\mathrm{n})$ and any matrix $A$ is brought back to the identity matrix choosing $L=A^{-1 / 2}$ with $A^{1 / 2}$ defined by the polar factor $R^{\prime}$ in (1). Likewise, any tangent vector $X \in \operatorname{Sym}(\mathrm{n})$ at identity $I \in \mathrm{P}_{\mathrm{n}}$ can be transported to a tangent vector $A^{1 / 2} X A^{1 / 2} \in T_{A} P_{n}$. At identity $I$, the $\mathrm{Gl}(\mathrm{n})$-invariant metric is defined as the usual scalar product $g_{I}^{P_{n}}\left(X_{1}, X_{2}\right)=\operatorname{Tr}\left(X_{1} X_{2}^{T}\right)=$ $\operatorname{Tr}\left(X_{1} X_{2}\right)$. The invariance of the metrics then implies $g_{A}^{P_{n}}\left(A^{1 / 2} X_{1} A^{1 / 2}, A^{1 / 2} X_{2} A^{1 / 2}\right)=$ $\operatorname{Tr}\left(X_{1} X_{2}\right)$, which can only be satisfied with the definition

$$
g_{A}^{P_{n}}\left(D_{1}, D_{2}\right)=\operatorname{Tr}\left(D_{1} A^{-1} D_{2} A^{-1}\right)
$$

at any arbitrary $A \in \mathrm{P}_{\mathrm{n}}$. The invariance of the metric (4) has direct implications on the expression of the geodesics and the accompanying Riemannian distance. The exponential map at $I$ is the usual matrix exponential

$$
\exp _{I}^{P_{n}} X=\exp X=\sum_{k=0}^{\infty}(1 / k !) X^{k} .
$$

The Frobenius norm $\|X\|_{F}$ is the geodesic length $d(\exp X, I)=\|X\|_{F}$, hence the formula at identity

$$
d(A, I)=\|\log A\|_{F} .
$$

Invariance of the metric again extends the characterization of geodesics at arbitrary $A \in \mathrm{P}_{\mathrm{n}}[21,32]$ :

$$
\exp _{A}^{P_{n}}(t X)=A^{1 / 2} \exp \left(t A^{-1 / 2} X A^{-1 / 2}\right) A^{1 / 2}, \quad t>0,
$$


and the corresponding geodesic distance

$$
d_{P_{n}}(A, B)=d\left(A^{-1 / 2} B A^{-1 / 2}, I\right)=\left\|\log \left(A^{-1 / 2} B A^{-1 / 2}\right)\right\|_{F}=\sqrt{\sum_{k} \log ^{2}\left(\lambda_{k}\right)}
$$

where $\lambda_{k}$ are the generalized eigenvalues of the pencil $A-\lambda B$, i.e., the roots of $\operatorname{det}\left(A B^{-1}-\lambda I\right)$. Note that the distance is invariant with respect to matrix inversion $(A, B) \mapsto\left(A^{-1}, B^{-1}\right)$ because $\log ^{2}\left(\lambda_{k}\right)$ is invariant to inversion $\lambda_{k} \mapsto \lambda_{k}^{-1}$.

The geodesic characterization provides a closed-form expression of the Riemannian (Karcher) mean of two matrices $A, B \in \mathrm{P}_{\mathrm{n}}$. The geodesic $A(t)$ linking $A$ and $B$ is

$$
A(t)=\exp _{A}^{P_{n}}(t X)=A^{1 / 2} \exp \left(t \log \left(A^{-1 / 2} B A^{-1 / 2}\right)\right) A^{1 / 2},
$$

where $A^{-1 / 2} X A^{-1 / 2}=\log \left(A^{-1 / 2} B A^{-1 / 2}\right) \in \operatorname{Sym}(\mathrm{n})$. The midpoint is obtained for $t=1 / 2$ :

$$
A \circ B=A^{1 / 2}\left(A^{-1 / 2} B A^{-1 / 2}\right)^{1 / 2} A^{1 / 2} .
$$

For a comprehensive treatment of the geometric versus arithmetic means of positive definite matrices, the reader is referred to $[4,21]$. From a geometric viewpoint, there are numerous reasons to prefer the natural metric (and associated distance and mean) on the symmetric cone to the flat metric associated to the distance $\|A-B\|_{F}$. Indeed the symmetric cone is not a vector space. The flat metric can still be used because the set is convex. However, many applications treating the space of covariance matrices as a vector space can yield to degraded algorithm performances [32]. A further undesirable feature of the flat metric is that $\mathrm{P}_{\mathrm{n}}$ is not a geodesically complete space (see section 5) since the geodesic $A+t(B-A)$ is not a positive matrix for all $t$. In contrast, it becomes geodesically complete with the natural metric. A practical consequence is that the natural metric is well-suited to short-step interior point methods in $P_{n}$. Remarkably, it coincides with the metric defined by the natural self-concordant logarithmically homogeneous barrier (-log det $A$ ) on the symmetric cone (which is a convex set) in optimization $[9,22]$. Furthermore, the invariance to the group action (3) (implying in particular invariance with respect to inversion) is desirable in numerous applications (see, e.g., $[6,26,32,7])$. In particular, if $A$ is a covariance matrix $\mathbb{E}\left(z z^{T}\right)$, the action corresponds to a change of basis $z \mapsto L z$. As a consequence the natural metric is well-suited to intrinsic estimation algorithms for covariance matrix estimation; see [32]. In [26, 14, 6], the authors argue that invariance 
with respect to inversion is adapted to the physics of diffusion tensors related to medical imaging. Finally, the associated (geometric) mean has numerous desirable properties developed in section 6 . It is useful to keep in mind that the geometric mean coincides with the classical geometric mean $\sqrt{a b}$ when $a, b \in \mathrm{P}_{1}$.

\section{Extending the metric: A geometric insight in the plane}

In order to inherit some of the nice properties of the natural distance on $\mathrm{P}_{\mathrm{n}}$ when the matrices are not full-rank, we seek a distance which is invariant to the $\mathrm{Gl}(\mathrm{n})$ group action. For tutorial purposes we start by considering $2 \times 2$ matrices of rank 1: $\mathrm{S}^{+}(1,2)$. For any $L \in \mathrm{Gl}(2)$ and $A, B \in \mathrm{S}^{+}(1,2)$ one wants

$$
d(A, B)=d\left(L A L^{T}, L B L^{T}\right) .
$$

This objective is, however, too ambitious. Taking

$$
A=\left(\begin{array}{ll}
1 & 0 \\
0 & 0
\end{array}\right), \quad B=\left(\begin{array}{ll}
1 & 1 \\
1 & 1
\end{array}\right), \quad \text { and } \quad L=\left(\begin{array}{ll}
1 & 0 \\
0 & \epsilon
\end{array}\right)
$$

$A$ is unchanged by the transformation whereas $L B L^{T}=\left(\begin{array}{cc}1 & \epsilon \\ \epsilon & \epsilon^{2}\end{array}\right)$. Thus if the distance is continuous in the matrix elements (for example it is associated to a metric), we have in the limit $d(A, B)=d(A, A)=0$ as $\epsilon \rightarrow 0$. This proves that $d$ is not a distance since $A \neq B$.

Mimicking the matrix decomposition (1), write $A \in \mathrm{S}^{+}(1,2)$ :

$$
A=x x^{T}=u r^{2} u^{T}
$$

where $u=(\cos (\theta), \sin (\theta)) \in \mathbb{S}^{1} \subset \mathbb{R}^{2}$ is a unit vector and $(r, \theta)$ is the polar representation of $x$. Without loss of generality $\theta$ is equated to $\theta+j \pi$, $j \in \mathbb{Z}$, since $x$ and $-x$ correspond to the same $A$. Thus $\mathrm{S}^{+}(1,2)$ can be equated to the space $\mathbb{R}_{*}^{+} \times \mathbb{R P}^{1}$, where $\mathbb{R P}^{1}$ is the real projective space of dimension 1 (lines of $\mathbb{R}^{2}$ ). The group action (3) corresponds to a change of basis for the vector $x \mapsto L x$. We have proved in the last paragraph that there is no distance which is invariant under every change of basis for $x$. Nevertheless one can define a distance which is invariant by scaling and orthogonal transformation, i.e., $G=\{\mu R:(\mu, R) \in \mathbb{R} \times O(2)\}$. A sensible metric is $d s^{2}=d \theta^{2}+k(d r / r)^{2}=d \theta^{2}+k d(\log r)^{2}$ with $k>0$. Let us find the distance associated to this metric. Let $A=x_{1} x_{1}^{T}$ and $B=x_{2} x_{2}^{T}$. Let 
$\left(r_{i}, \theta_{i}\right)$ denote their polar coordinates for $i=1,2$. It is always possible to have $\left|\theta_{2}-\theta_{1}\right| \leq \pi / 2$ possibly replacing $\theta_{1}$ by $\theta_{1} \pm \pi$. The distance is then

$$
d_{\mathrm{S}^{+}(1,2)}^{2}(A, B)=\left|\theta_{2}-\theta_{1}\right|^{2}+k\left|\log \left(r_{1} / r_{2}\right)\right|^{2}, \quad k>0 .
$$

where the first term penalizes the distance between the subspaces range $(A)=$ $\operatorname{span}\left(x_{1}\right)$ and range $(B)=\operatorname{span}\left(x_{2}\right)$. It ensures the invariance by rotation/orthogonal transformation (which only affects $\theta$ ) while the second term ensures the invariance by scaling (which only affects $r$ ). Note that when $\operatorname{range}(A)=\operatorname{range}(B)$ the induced distance on the 1-dimensional subspace corresponds to the natural distance (5) between matrices of $P_{1}$. The metric above rewrites in the general form

$$
d s^{2}=\operatorname{Tr}\left(d u^{T} d u\right)+k d(\log r)^{2}, \quad k>0 .
$$

A symmetry-based justification The choice of the metric (8) can be derived from necessary conditions imposed by the desired symmetries (invariances). Let $A=x_{1} x_{1}^{T}, B=x_{2} x_{2}^{T}$ with $x_{1}, x_{2} \in \mathbb{R}^{2}$. We seek an invariant distance between $A$ and $B$. This is equivalent to seeking an invariant distance between $x_{1}$ and $x_{2}$; i.e., a scalar $G$-invariant function on $\mathbb{R}^{2} \times \mathbb{R}^{2}$, satisfying the conditions required to be a distance. If $H$ is any subgroup of $G$, the distance must be in particular $H$-invariant. Let $H=\mathbb{R}_{*}^{+} \times S O(2) \subset G$. The identification $\mathbb{R}_{*}^{2} \cong H$ can be obtained via polar coordinates $(r, \theta) \in \mathbb{R}_{*}^{+} \times S^{1} \cong H$. A standard result (see, e.g., $[23,8]$ ) is that every $H$-invariant scalar function of $\left(x_{1}, x_{2}\right) \in H \times H$ (in particular any distance) is a function of the scalar invariants $\left(r_{2} / r_{1}, \theta_{2}-\theta_{1}\right)$; i.e., the coordinates of $x_{1}^{-1} * x_{2}$, where $*$ is the group multiplication of $H$. Thus any invariant distance $d$ writes $d\left(x_{1}, x_{2}\right)=h\left(r_{2} / r_{1}, \theta_{2}-\theta_{1}\right)$, and since $d$ is a distance it must be symmetric $d\left(x_{1}, x_{2}\right)=d\left(x_{2}, x_{1}\right)$. Thus necessarily $d\left(x_{1}, x_{2}\right)=f\left(\left|\log \left(r_{2} / r_{1}\right)\right|,\left|\theta_{2}-\theta_{1}\right|\right)$ with $f$ positive, monotone in each of its arguments, and only $f(0,0)$ is equal to 0 . It proves that the distance $(7)$ is a prototype for every $G$-invariant distance $d(A, B)$.

A physical justification The invariance properties of the distance (7) are meaningful from a physical viewpoint. Suppose $Z=\mathbb{E}\left(z z^{T}\right)$ is the covariance matrix of a stochastic variable $z \in \mathbb{R}^{2}$ (for instance the position of an object) with zero mean such that every realization of $z$ is on a line. Then $Z \in \mathrm{S}^{+}(1,2)$. The distance ( 7 ) between the two covariances $Z_{1}, Z_{2}$ of two independent variables $z_{1}, z_{2}$ (dispersing in two different directions) is well-defined. It does not depend on any nontrivial way on the choice of measurement units, e.g., feet versus meters, as well as on the orientation of the frame chosen, e.g., the first axis is pointing north or south. 
Moreover, suppose the measurements are noisy. For instance $Z_{1}=\operatorname{diag}\left(4, \epsilon^{2}\right)$ and $Z_{2}=\operatorname{diag}\left(\epsilon^{2}, 1\right)$, where the term $\epsilon \ll 1$ is the amplitude of the covariance of the noise. These two matrices belong to $P_{2}$, and the geometric mean (6) is $\operatorname{diag}(2 \epsilon, \epsilon)$. The smallness of the noise ruins the mean, which no longer reflects the physical interpolation between the two processes. Indeed in the degenerate case $\epsilon \rightarrow 0$, the mean becomes the null matrix. In contrast, the midpoint in the sense of the distance (7) between the rank 1 approximations of the two matrices (i.e., $\epsilon=0)$ is $\left(\begin{array}{ll}1 & 1 \\ 1 & 1\end{array}\right)$ with eigenvalues $(0,2)$, which is an image of the covariance matrix of the midpoint. The distance between the matrices and their mean is independent of the choice of units and orientation, and is hardly affected by noise.

\section{A new Riemannian metric on the symmet- ric semidefinite cone}

Let $\mathrm{S}^{+}(\mathrm{p}, \mathrm{n})$ be the set of positive semidefinite matrices of fixed-rank $p<n$. One can prove (analogously to the last section) in the general case that is impossible to find a distance between matrices of $\mathrm{S}^{+}(\mathrm{p}, \mathrm{n})$ which would be invariant to the transformation (3) for $p$ arbitrary small. Nevertheless one can construct a metric generalizing (8) and a distance generalizing (7). The Grassmanian distance is a multidimensional generalization of the angular distance $d \theta$, and the natural metric on the symmetric cone (4) is a multidimensional generalization of $d \log r=r^{-1} d r$. The resulting metric is invariant to orthogonal transformations and scalings. Locally, when the ranges of two infinitely close matrices coincide, the induced metric on the corresponding subspace reduces to the natural metric on the symmetric cone $\mathrm{P}_{\mathrm{p}}$. In particular, when $p=n$ the metric coincides with the natural metric on $\mathrm{P}_{\mathrm{n}}$.

Mimicking the developments of section 2, we start from the matrix equalities

$$
A=Z Z^{T}=(U R)(U R)^{T}=U R^{2} U^{T},
$$

where $A \in \mathrm{S}^{+}(\mathrm{p}, \mathrm{n}), Z \in \mathbb{R}_{*}^{n \times p}, R \in \mathrm{P}_{\mathrm{p}}, U \in \mathrm{V}_{\mathrm{n}, \mathrm{p}}$. Right multiplication of $Z$ by an orthogonal matrix $Z \mapsto Z O, O \in \mathrm{O}(\mathrm{p})$, does not affect the product $A=Z Z^{T}$. Consider the following $\mathrm{O}(\mathrm{p})$ group action:

$$
\begin{aligned}
& R \mapsto O^{T} R O \quad \in \mathrm{P}_{\mathrm{p}}, \\
& U \mapsto U O \quad \in \mathrm{V}_{\mathrm{n}, \mathrm{p}} .
\end{aligned}
$$

The representation $A=U R^{2} U^{T}$ with $\left(U, R^{2}\right) \in \mathrm{V}_{\mathrm{n}, \mathrm{p}} \times \mathrm{P}_{\mathrm{p}}$ is thus univocal up to the equivalence relation $\left(U, R^{2}\right) \equiv\left(U O, O^{T} R^{2} O\right)$ for any $O \in \mathrm{O}(\mathrm{p})$. Thus 
the set $\mathrm{S}^{+}(\mathrm{p}, \mathrm{n})$ admits a quotient manifold representation

$$
\mathrm{S}^{+}(\mathrm{p}, \mathrm{n}) \cong\left(\mathrm{V}_{\mathrm{n}, \mathrm{p}} \times \mathrm{P}_{\mathrm{p}}\right) / \mathrm{O}(\mathrm{p}) .
$$

(A dimension checking yields that the dimension of $\mathrm{S}^{+}(\mathrm{p}, \mathrm{n})$ is $\operatorname{dim}\left(\mathrm{V}_{\mathrm{n}, \mathrm{p}} \times \mathrm{P}_{\mathrm{p}}\right)$ $\operatorname{dim}(\mathrm{O}(\mathrm{p}))=p n-p(p-1) / 2$.) Note that the chosen quotient geometry differs from the one recently considered in [3], where elements of $\mathrm{S}^{+}(\mathrm{p}, \mathrm{n})$ are represented by equivalences classes $Z O, O \in \mathrm{O}(\mathrm{p})$, leading to the quotient representation $\mathrm{S}^{+}(\mathrm{p}, \mathrm{n}) \cong \mathbb{R}_{*}^{n \times p} / \mathrm{O}(\mathrm{p})$.

If $\left(U, R^{2}\right) \in \mathrm{V}_{\mathrm{n}, \mathrm{p}} \times \mathrm{P}_{\mathrm{p}}$ represents $A \in \mathrm{S}^{+}(\mathrm{p}, \mathrm{n})$, it is tempting to represent the tangent vectors of $T_{A} \mathrm{~S}^{+}(\mathrm{p}, \mathrm{n})$ by the infinitesimal variation $(\Delta, D)$, where

$$
\begin{aligned}
& \Delta=U_{\perp} B, \quad B \in \mathbb{R}^{(n-p) \times p}, \\
& D=R D_{0} R
\end{aligned}
$$

such that $U_{\perp} \in V_{n, n-p}, U^{T} U_{\perp}=0$, and $D_{0} \in \operatorname{Sym}(\mathrm{p})=T_{I} \mathrm{P}_{\mathrm{p}}$. The chosen metric of $\mathrm{S}^{+}(\mathrm{p}, \mathrm{n})$ is merely the sum of the infinitesimal distance in $\operatorname{Gr}(\mathrm{p}, \mathrm{n})$ and in $\mathrm{P}_{\mathrm{p}}$ :

$g_{\left(U, R^{2}\right)}\left(\left(\Delta_{1}, D_{1}\right),\left(\Delta_{2}, D_{2}\right)\right)=\operatorname{Tr}\left(\Delta_{1}^{T} \Delta_{2}\right)+k \operatorname{Tr}\left(R^{-1} D_{1} R^{-2} D_{2} R^{-1}\right), \quad k>0$,

generalizing (4) in a natural way. The next theorem proves that the construction endows the space $\mathrm{S}^{+}(\mathrm{p}, \mathrm{n})$ with a Riemannian structure.

Theorem 1 The space $\mathrm{S}^{+}(\mathrm{p}, \mathrm{n}) \cong\left(\mathrm{V}_{\mathrm{n}, \mathrm{p}} \times \mathrm{P}_{\mathrm{p}}\right) / \mathrm{O}(\mathrm{p})$ endowed with the metric (10) is a Riemannian manifold with horizontal space

$$
\mathcal{H}_{\left(U, R^{2}\right)}=\left\{(\Delta, D): \Delta=U_{\perp} B, B \in \mathbb{R}^{(n-p) \times p}, D=R D_{0} R, D_{0} \in \operatorname{Sym}(\mathrm{p})\right\} .
$$

Furthermore, the metric is invariant with respect to orthogonal transformations, scalings, and pseudoinversion.

Proof: In this proof we also recap some results on quotient manifolds. We follow the machinery of Riemannian quotient manifold (see, e.g., [2]), except that we will not require that the quotient map be a Riemannian submersion. Any representative $\left(U, R^{2}\right)$ lives in the structure space $\mathrm{V}_{\mathrm{n}, \mathrm{p}} \times \mathrm{P}_{\mathrm{p}}$. The tangent space to $\left(U, R^{2}\right)$ is the direct sum of a vertical space and a horizontal space: $T_{\left(U, R^{2}\right)}\left(\mathrm{V}_{\mathrm{n}, \mathrm{p}} \times \mathrm{P}_{\mathrm{p}}\right)=\mathcal{V}_{\left(U, R^{2}\right)} \oplus \mathcal{H}_{\left(U, R^{2}\right)}$. The equivalence class $\left\{\left(U O, O^{T} R^{2} O\right), O \in \mathrm{O}(\mathrm{p})\right\}$ is called a fiber. The vertical space is the tangent space to the fiber at $\left(U, R^{2}\right)$ :

$$
\mathcal{V}_{\left(U, R^{2}\right)}=\left\{\left(U \Omega, R^{2} \Omega-\Omega R^{2}\right): \Omega \in T_{I} \mathrm{O}(\mathrm{p})\right\}
$$


i.e., $\Omega$ is a skew-symmetric matrix. The horizontal space $\mathcal{H}_{\left(U, R^{2}\right)}$ is by definition complementary to $\mathcal{V}_{\left(U, R^{2}\right)}$. It is customary to represent tangent vectors to the quotient manifold only as elements of the horizontal space. Indeed it is useless to consider elements of the vertical space since they are tangent to the fiber, and all elements of the fiber represent the same point in the quotient manifold. Here the tangent space to the product manifold writes $T_{\left(U, R^{2}\right)}\left(\mathrm{V}_{\mathrm{n}, \mathrm{p}} \times \mathrm{P}_{\mathrm{p}}\right)=\left\{(\Xi, D): \quad \Xi \in T_{U} \mathrm{~V}_{\mathrm{n}, \mathrm{p}}, D \in T_{R^{2}} \mathrm{P}_{\mathrm{p}}\right\}$. So $\Xi=\Delta+U \Omega$ with $\Omega$ skew-symmetric and $\Delta=U_{\perp} B$. But $\left(U \Omega, R^{2} \Omega-\Omega R^{2}\right) \in \mathcal{V}_{(U, M)}$. So the horizontal space at $T_{\left(U, R^{2}\right)}$ can be chosen to be made only of the vectors $(\Delta, D)$ given by $(9)$ :

$$
\mathcal{H}_{\left(U, R^{2}\right)}=\left\{(\Delta, D): \Delta=U_{\perp} B, B \in \mathbb{R}^{(n-p) \times p}, D=R D_{0} R, D_{0} \in \operatorname{sym}(p)\right\} .
$$

The manifold $\mathrm{S}^{+}(\mathrm{p}, \mathrm{n})$ endowed with the metric (10) is a Riemannian quotient manifold: after having chosen a Riemannian metric on the structure space, all we must prove is that the induced metric on the horizontal space does not depend on the representative chosen. The Riemannian metric for the product manifold (structure space) $\mathrm{V}_{\mathrm{n}, \mathrm{p}} \times \mathrm{P}_{\mathrm{p}}$ can be chosen as the sum of the natural metrics of $\mathrm{V}_{\mathrm{n}, \mathrm{p}}$ and $\mathrm{P}_{\mathrm{p}}: g_{\left(U, R^{2}\right)}^{S P}\left(X_{1}, X_{2}\right)=\operatorname{Tr}\left(\Omega_{1}^{T} \Omega_{2}+\Delta_{1}^{T} \Delta_{2}\right)+$ $k \operatorname{Tr}\left(D_{1} R^{-2} D_{2} R^{-2}\right)$, where $X_{i}=\left(U \Omega_{i}+\Delta_{i}, D_{i}\right)$ for $i=1,2$. Let $X_{1}, X_{2} \in$ $\mathcal{H}_{\left(U, R^{2}\right)}$. We have $g_{\left(U, R^{2}\right)}^{S P}\left(X_{1}, X_{2}\right)=\operatorname{Tr}\left(\Delta_{1}^{T} \Delta_{2}\right)+k \operatorname{Tr}\left(D_{1} R^{-2} D_{2} R^{-2}\right)$. The first term only depends on $U_{\perp}$, which is invariant along the fiber, and the second term is $\operatorname{Tr}\left(D_{0,1} D_{0,2}\right)$, where $D_{i}=R D_{0, i} R$, and it does not depend on the representative chosen. These invariances are due indeed to the invariance of the Grassman metric with respect to the representative in the Stiefel manifold, and the invariance properties of the natural metric on $\mathrm{P}_{\mathrm{p}}$.

Concerning the last point of the theorem, an orthogonal transformation affects the representative $\left(U, R^{2}\right)$ by transforming $U$ in $O U$ with $O \in \mathrm{O}(\mathrm{n})$ and thus $\Delta$ in $O \Delta$; and a scaling by transforming $R^{2}$ in $\mu^{2} R^{2}$ with $\mu \in \mathbb{R}_{*}$ and thus $D$ in $\mu^{2} D$. Thus they affect separately the first and second term of the metric, which are both invariant to each of these transformations. The invariance to pseudoinversion derives from the invariance to inversion of the natural metric on $\mathrm{P}_{\mathrm{p}}$, as $\left(U, R^{-2}\right)$ is a representative of a pseudoinverse of $U R^{2} U^{T}$. These properties are further detailed in the proof of Theorem 2 .

The reader will note that with the proposed choice of metric and horizontal space, $\mathcal{V}_{\left(U, R^{2}\right)}$ and $\mathcal{H}_{\left(U, R^{2}\right)}$ are complementary, but not orthogonal. This is rather unusual, and it will have implications in the sequel (Proposition 1). However, they tend to be orthogonal as $k \rightarrow 0$.

As a concluding remark for this section, we recall that the natural metric on the cone is not the only GL(n)-invariant metric, and a whole family of invariant metrics can be derived from the scalar product at identity 
$\operatorname{Tr}\left(D_{1}^{T} D_{2}\right)+\beta \operatorname{Tr}\left(D_{1}\right) \operatorname{Tr}\left(D_{2}\right)$, with $\beta>-\frac{1}{n}$ (see Appendix 7.2). For instance, the metric corresponding to $\beta=-\frac{1}{n+1}$ was proposed in [20] as a Riemannian metric on the space of Gaussian distributions having a nonzero mean. This family of metrics could also easily be extended to $\mathrm{S}^{+}(\mathrm{p}, \mathrm{n})$. It should be clear that the results of Theorem 1 hold for any GL(n)-invariant metric on the cone and not only for the natural one.

\section{$5 \quad$ Horizontal geodesics in the structure space approximate geodesics in $\mathrm{S}^{+}(\mathbf{p}, \mathbf{n})$}

This section provides the construction of special curves of interest connecting any two matrices $A$ and $B$ in $\mathrm{S}^{+}(\mathrm{p}, \mathrm{n})$ : horizontal geodesics in the structure space. First of all let us find two representatives of $A$ and $B$ in $\mathrm{V}_{\mathrm{n}, \mathrm{p}} \times \mathrm{P}_{\mathrm{p}}$ connected by a horizontal geodesics. Let $V_{A}, V_{B} \in \mathrm{V}_{\mathrm{n}, \mathrm{p}}$ be two matrices that span range $(A)$ and $\operatorname{range}(B)$, respectively. The SVD of $V_{B}^{T} V_{A}$ yields $O_{A}, O_{B} \in \mathbb{R}^{p \times p}$ such that

$$
O_{A}^{T} V_{A}^{T} V_{B} O_{B}=\operatorname{diag}\left(\sigma_{1}, \cdots, \sigma_{p}\right), \quad 1 \geq \sigma_{1} \geq \cdots \geq \sigma_{p} \geq 0
$$

The $\sigma_{i}=\cos \theta_{i}$ are the cosines of the principal angles $0 \leq \theta_{1} \leq . . \leq$ $\theta_{p} \leq \pi / 2$ between the two subspaces [15]. Choosing the principal vectors $U_{A}=\left(u_{1}^{A}, \cdots, u_{p}^{A}\right)=V_{A} O_{A}$ and $U_{B}=\left(u_{1}^{B}, \cdots, u_{p}^{B}\right)=V_{B} O_{B}$ yields a simple formula for the Grassman geodesic connecting range $(A)$ and $\operatorname{range}(B)$ (e.g. $[32])$ :

$$
U(t)=U_{A} \cos (\Theta t)+X \sin (\Theta t)
$$

where $\Theta=\operatorname{diag}\left(\theta_{1}, \cdots, \theta_{p}\right)$ and $X$ is the normalized projection of $V$ onto the column space of $U_{\perp}$, i.e., $X=\left(I-U_{A} U_{A}^{T}\right) U_{B} F$ where $F$ is the pseudoinverse of the matrix $\operatorname{diag}\left(\sin \left(\theta_{1}\right), \cdots, \sin \left(\theta_{p}\right)\right)$. The associated geodesic $R^{2}(t)$ in $\mathrm{P}_{\mathrm{p}}$ must connect $R_{A}^{2}:=U_{A}^{T} A U_{A}$ and $R_{B}^{2}:=U_{B}^{T} B U_{B}$, that is,

$$
R^{2}(t)=R_{A} \exp \left(t \log R_{A}^{-1} R_{B}^{2} R_{A}^{-1}\right) R_{A} .
$$

Theorem 2 The singular value decomposition (11) and the geodesic curves (12) and (13) define a curve in $\mathrm{S}^{+}(\mathrm{p}, \mathrm{n})$

$$
\gamma_{A \rightarrow B}(t)=U(t) R^{2}(t) U^{T}(t)
$$

with the following properties:

- $\gamma_{A \rightarrow B}(\cdot)$ connects $A$ and $B$ in $\mathrm{S}^{+}(\mathrm{p}, \mathrm{n})$, that is, $\gamma_{A \rightarrow B}(0)=A, \gamma_{A \rightarrow B}(1)=$ $B$, and $\gamma_{A \rightarrow B}(t) \in \mathrm{S}^{+}(\mathrm{p}, \mathrm{n}) \forall t \in[0,1]$. 
- The curve $\left(U(t), R^{2}(t)\right)$ is a horizontal lift of $\gamma_{A \rightarrow B}(t)$ and it is a geodesic in the structure space $V_{n, p} \times P_{p}$.

- The (squared) total length of $\gamma_{A \rightarrow B}(t)$ in the Riemannian manifold $\left(\mathrm{S}^{+}(\mathrm{p}, \mathrm{n}), \mathrm{g}\right)$ is given by

$$
l^{2}\left(\gamma_{A \rightarrow B}\right)=\|\Theta\|_{F}^{2}+k\left\|\log R_{A}^{-1} R_{B}^{2} R_{A}^{-1}\right\|_{F}^{2} .
$$

It is invariant with respect to pseudoinversion and to the group action by congruence of orthogonal transformations and scalings.

Furthermore, the curve $\gamma_{A \rightarrow B}(\cdot)$ is uniquely defined provided that the $(p-1)$ th principal angle satisfies $\theta_{p-1} \neq \pi / 2$.

The proof of the theorem is given in Appendix 7.2. Viewing matrices of $\mathrm{S}^{+}(\mathrm{p}, \mathrm{n})$ as flat ellipsoids in $\mathbb{R}^{n}$, the length $l\left(\gamma_{A \rightarrow B}\right)$ consists of two independent contributions: a distance between the subspaces in which the ellipsoids are contained (Grassman distance), and a distance between the ellipsoids within a common subspace (natural distance on the cone). In this sense, (15) provides an exact generalization of the Riemannian distance (7) of Section 3. However, it is not the Riemannian distance of $\left(\mathrm{S}^{+}(\mathrm{p}, \mathrm{n}), \mathrm{g}\right)$ because $\gamma_{A \rightarrow B}(\cdot)$ is not necessarily a geodesic curve (even tough it is the base curve of a horizontal geodesic in the structure space).

Proposition 1 The curve $\gamma_{A \rightarrow B}(\cdot)$ is not necessarily a geodesic of $\left(\mathrm{S}^{+}(\mathrm{p}, \mathrm{n}), \mathrm{g}\right)$. Its length provides a meaningful measure of closeness between $A$ and $B$ which is not a distance because it does not satisfy the triangle inequality.

A proof of this proposition is given by the following example: Let $A=$ $\operatorname{diag}(2,1,0)$ and $B=\operatorname{diag}(1,2,0)$. Because $A$ and $B$ have the same range, the length $l\left(\gamma_{A \rightarrow B}\right)$ reduces to a distance in the cone $P_{2}$ (no Grassman contribution). But $A$ can also be connected to $B$ via $C=\operatorname{diag}(0,1,2)$ and $D=$ $\operatorname{diag}(1,0,2)$. The curves $\gamma_{A \rightarrow C}(\cdot), \gamma_{C \rightarrow D}(\cdot)$, and $\gamma_{D \rightarrow B}(\cdot)$ each involve a pure subspace rotation of $\pi / 2$, which means that their total length in $\left(\mathrm{S}^{+}(\mathrm{p}, \mathrm{n}), \mathrm{g}\right)$ is $3 \pi / 2$ (no contribution in the cone). The situation $l\left(\gamma_{A \rightarrow B}\right)>3 \pi / 2$ is clearly possible if $k$ is large enough, showing that $\gamma_{A \rightarrow B}(\cdot)$ is not necessarily a geodesic curve. Even if the triangular inequality is not satisfied, the proposed measure of closeness satisfies the two other properties of a distance: it is symmetric, and vanishes only when the two matrices coincide.

The construction of the curve $\gamma_{A \rightarrow B}(\cdot)$ has a geometrical meaning in $\mathrm{S}^{+}(\mathrm{p}, \mathrm{n})$ : If a gyroscope is attached to the moving ellipsoid represented by $U(t) R_{A}^{2} U(t)$, where $U(t)$ is given by (12), it indicates no rotation around an axis perpendicular to the ellipsoid during the motion $0 \leq t \leq 1$. Thus the ellipsoid $U(1) R_{A}^{2} U(1)=U_{B} R_{A}^{2} U_{B}$ is the ellipsoid $A=U(0) R_{A}^{2} U(0)=U_{A} R_{A}^{2} U_{A}$ 
brought in range $(B)$ by a rotation of minimal energy. This also justifies comparing directly $R_{A}^{2}$ to $R_{B}^{2}$ in $\mathrm{P}_{\mathrm{p}}$. If $O \neq I$ is an orthogonal matrix, it would violate the rotational invariance to compare $R_{A}^{2}$ to $O^{T} R_{B}^{2} O$, even though $\left(U_{B} O, O^{T} R_{B} O\right)$ is a valid representation of $B$ and the distance in Grassman between range $\left(U_{A}\right)$ and range $\left(U_{B}\right)$ is unaffected by the transformation $U_{B} \mapsto U_{B} O$.

Even tough it is not a distance, the closeness $l\left(\gamma_{A \rightarrow B}\right)$ is a meaningful generalization of the Riemannian distance (7) of Section 3. It reduces to the natural distance in the cone when $A$ and $B$ span the same subspace and it reduces to the Grassmann Riemannian distance between range $(A)$ and range $(B)$ when $A$ and $B$ are rank $p$ projectors. Furthermore, it recovers a maximal number of the desirable invariance properties of the natural distance on the symmetric cone, exactly as expected from the planar example in Section 3. Homothetic transformations, isometries leaving the origin fixed, and pseudoinversion correspond to angle-preserving transformations (when $n>2$, these transformations are the conformal transformations of the Euclidean space). Note we already proved in Section 3 that the invariance with respect to transformations which do not preserve the angles is anyway an impossible property to obtain, at least when $p$ is small enough.

The computation of the closeness (15) involves the computation of principal angles and vectors, which is standard, and can be done via QR factorization at a numerical cost $O\left(n p^{2}\right)$. The computation of the second term in (15) involves a symmetric generalized eigenvalue problem, which requires $O\left(p^{3}\right)$ operations. The linear complexity in the dimension $n$ makes the distance calculation efficient even in large-scale problems provided $p \ll n$.

The following proposition establishes a link between the closeness measure $l$ and the Riemannian distance $d_{\mathrm{S}^{+}(\mathrm{p}, \mathrm{n})}$ in $\left(\mathrm{S}^{+}(\mathrm{p}, \mathrm{n}), g\right)$.

Proposition 2 Let $A=U_{A} R_{A}^{2} U_{A}^{T}$ and $B=U_{B} R_{B}^{2} U_{B}^{T}$ be two elements of $\mathrm{S}^{+}(\mathrm{p}, \mathrm{n})$. We have

$$
0 \leq l^{2}\left(\gamma_{A \rightarrow B}\right)-d_{\mathrm{S}^{+}(\mathrm{p}, \mathrm{n})}^{2}(A, B) \leq k \max _{O \in O(p)} d_{\mathrm{P}_{\mathrm{p}}}^{2}\left(R_{B}^{2}, O^{T} R_{B}^{2} O\right) .
$$

The right term is well defined for all $O \in O(p)$, and it is finite since $O(p)$ is a compact set. Moreover it tends to 0 as $k \rightarrow 0$. Thus the curves defined above are always longer than the geodesics, but they yield good approximations of the geodesics when the Grassman contribution is highly penalized, i.e., $k$ is small (proof in Appendix 7.2).

We conclude this section by observing that horizontal geodesics provide a complete family of line search curves in $\mathrm{S}^{+}(\mathrm{p}, \mathrm{n})$. The curve emanating from $A=U R^{2} U^{T}$ in the direction $(\Delta, D) \in \mathcal{H}_{\left(U, R^{2}\right)}$ admits the analytical 
characterization

$$
\gamma_{A}(t)=U(t) R^{2}(t) U^{T}(t)
$$

where

$$
U(t)=U V^{T} \cos (\Gamma t) V+X \sin (\Gamma t) V
$$

is the (Grassman geodesic) curve emanating from $U$ in direction $\Delta$, i.e. $X \Gamma V=\Delta$ is the compact SVD of $\Delta ;$ and

$$
R^{2}(t)=R \exp \left(t R^{-1} D R^{-1}\right) R
$$

is the $\left(\mathrm{P}_{\mathrm{p}}\right.$ geodesic) curve emanating from $R^{2}$ in the direction $D$. An interesting feature is that neither the curve $\gamma_{A}(\cdot)$ nor the geodesics reach the boundary of $\mathrm{S}^{+}(\mathrm{p}, \mathrm{n})$ in finite time (proof in Appendix 7.2).

Proposition 3 For any $A$ in $\mathrm{S}^{+}(\mathrm{p}, \mathrm{n})$, the curves $\gamma_{A}(\cdot)$ can be extended from $t=-\infty$ to $t=\infty$. Moreover, the manifold $\left(\mathrm{S}^{+}(\mathrm{p}, \mathrm{n}), g\right)$ is geodesically complete.

\section{Geometric mean in $\mathrm{S}^{+}(\mathrm{p}, \mathrm{n})$}

The closeness measure between $A$ and $B$ in the previous section provides a direct formula for the "halfway" matrix

$$
A \circ B=W K W^{T},
$$

where $K=R_{A}\left(R_{A}^{-1} R_{B}^{2} R_{A}^{-1}\right)^{1 / 2} R_{A}$ is the Riemannian mean of $R_{A}^{2}$ and $R_{B}^{2}$ in $\mathrm{P}_{\mathrm{p}}, W=\cos (\Gamma / 2) U_{A}+\sin (\Gamma / 2) X$ is the Riemannian mean of $\operatorname{range}(A)$ and range $(B)$, and where $R_{A}, R_{B}, U_{A}, X$ are defined via the $S V D$ (11).

The mean is uniquely defined in the generic case $\theta_{p} \neq \pi / 2$, as the mean in Grassman between range $(A)$ and $\operatorname{range}(B)$ is uniquely defined. When $\theta_{p}=\pi / 2$ and $\theta_{p-1} \neq \pi / 2$, according to Theorem $2 l\left(\gamma_{A \rightarrow B}\right)$ is uniquely defined, but there are two Riemannian means of range $(A)$ and range $(B)$ in Grassman. So the mean (17) is well-defined but there are two midpoints. If $\theta_{p-1}=\pi / 2$, there is an infinity of midpoints. Indeed, let $r$ be the number of principal angles equal to $\pi / 2$. Looking at the proof of Theorem 2 in Appendix 7.2, we see that the midpoint will necessarily be a matrix of the form (17) where $U_{A}, R_{A}$ are replaced by $U_{A} P, P^{T} R_{A} P$, with $P \in \mathrm{O}(\mathrm{p})$ representing an arbitrary rotation on the span of the $p-r$ last principal vectors. From now on we will systematically assume that $\theta_{p} \neq \pi / 2$. 
The geometric mean $A \circ B$ is closely related to the popular geometric mean $A \# B$ of Ando $[5,29,4]$. One definition of Ando mean is to consider $A \# B$ as the solution of the extremal problem

$$
\max \left\{X \succeq 0:\left(\begin{array}{cc}
A & X \\
X & B
\end{array}\right) \succeq 0\right\} .
$$

For full-rank positive definite matrices $A$ and $B$, the solution of (18) is given by $X=A^{1 / 2}\left(A^{-1 / 2} B A^{-1 / 2}\right)^{1 / 2} A^{1 / 2}$, that is, Ando mean coincides with the geometric mean (17). In contrast, for matrices $A$ and $B$ in $\mathrm{S}^{+}(\mathrm{p}, \mathrm{n})$, the geometric mean (17) differs from the Ando mean (18). In particular the geometric mean is by definition rank-preserving whereas the solution of (18) has a rank which is upper bounded by $\operatorname{dim}(\operatorname{range}(A) \cap \operatorname{range}(B))$. A simple example is provided by the example already discussed in Section 3. The geometric mean of $A=\operatorname{diag}(4,0)$ and $B=\operatorname{diag}(0,1)$ is $A \circ B=\left(\begin{array}{ll}1 & 1 \\ 1 & 1\end{array}\right)$ while the solution of (18) is $A \# B=\left(\begin{array}{ll}0 & 0 \\ 0 & 0\end{array}\right)$. This is easy to check because the Ando mean can be obtained by density: letting $A^{\prime}=A+\epsilon I$ and $B^{\prime}=B+\epsilon I$ the mean corresponds to the limit of $A^{\prime 1 / 2}\left(A^{\prime-1 / 2} B^{\prime} A^{\prime-1 / 2}\right)^{1 / 2} A^{\prime 1 / 2}$ for $\epsilon \rightarrow 0$.

Apart from this important difference, both the geometric mean (17) and the Ando mean enjoy most desirable properties of a matrix geometric mean [4], listed in the proposition below.

Proposition 4 The mean $A \circ B$ possesses the properties listed below.

i. Joint homogeneity $\alpha A \circ \beta B=(\alpha \beta)^{1 / 2} A \circ B$.

ii. Permutation invariance $A \circ B=B \circ A$.

iii. Monotonicity. If $A \leq A_{0}$ (i.e. $\left(A_{0}-A\right)$ is a positive matrix) and $B \leq B_{0}$, the means are comparable and verify $A \circ B \leq A_{0} \circ B_{0}$.

iv. Congruence invariance. For any $(\mu, P) \in \mathbb{R} \times \mathrm{O}\left(\right.$ n) we have $\left(\mu P^{T} A \mu P\right) \circ$ $\left(\mu P^{T} B \mu P\right)=\mu P^{T}(A \circ B) \mu P$.

v. Self-duality $(A \circ B)^{\dagger}=\left(A^{\dagger} \circ B^{\dagger}\right)$, where "†" denotes pseudoinversion.

The mean (17) may prove useful to generalize to low-rank positive semidefinite matrices the growing use of the geometric mean in applications requiring interpolation and the filtering of positive definite matrices; see e.g. $[26,6,7,34]$. 
A particular case of interest is the set of rank $p$ projectors

$$
\left\{P \in \mathbb{R}^{n \times n} / P^{T}=P, P^{2}=P, \operatorname{Tr}(P)=p\right\},
$$

which is in bijection with the Grassman manifold of $p$-dimensional subspaces. Not surprisingly, the geometric mean (17) of $A$ and $B$ in (19) agrees with the Riemannian mean of range $(A)$ and $\operatorname{range}(B)$ on $\operatorname{Gr}(p, n)$. On this set, the geometric mean also agrees with the Riemannian mean in $\mathrm{S}^{+}(\mathrm{p}, \mathrm{n})$ since $d_{\mathrm{S}^{+}(\mathrm{p}, \mathrm{n})}(A, B)=d_{\mathrm{Gr}(\mathrm{p}, \mathrm{n})}(\operatorname{range}(A), \operatorname{range}(B))$ for $A, B$ projectors (see Appendix 7.2).

On the other hand, when $A$ and $B$ have the same range, the geometric mean (17) does not necessarily agree with the Riemannian mean in $\mathrm{S}^{+}(\mathrm{p}, \mathrm{n})$ since the connecting curve $\gamma_{A \rightarrow B}(\cdot)$ is not necessarily a geodesic. Note however that the geometric mean is a good approximation of the Riemannian mean when $k$ is small. In fact the geometric mean (17) is a more natural extension of the Ando mean than the Riemannian mean since $A \circ B=U\left(R_{A}^{2} \# R_{B}^{2}\right) U^{T}$ for $A=U R_{A}^{2} U^{T}$ and $B=U R_{B}^{2} U^{T}$.

\section{Conclusion}

This paper generalizes the Riemannian geometry of the symmetric cone $\mathrm{P}_{\mathrm{n}}$ to the manifold $\mathrm{S}^{+}(\mathrm{p}, \mathrm{n})$ of positive semidefinite matrices of fixed-rank $p$. The generalization is based on the quotient geometry $\mathrm{S}^{+}(\mathrm{p}, \mathrm{n})=\left(\mathrm{V}_{\mathrm{n}, \mathrm{p}} \times \mathrm{P}_{\mathrm{p}}\right) / \mathrm{O}(\mathrm{p})$ that leads to a natural metric with decoupled contribution in $\operatorname{Gr}(\mathrm{p}, \mathrm{n})$ and the cone $\mathrm{P}_{\mathrm{p}}$. This geometry leads to an explicit and natural notion of closeness in $\mathrm{S}^{+}(\mathrm{p}, \mathrm{n})$, which in turn provides a computable and natural definition of Riemannian distance and rank-preserving geometric mean.

The proposed computational tools may prove useful in applications involving computations with low-rank approximations of large-scale positive definite matrices. Such applications have already appeared in MRI tensor computing [26, 25, 14, 6], and in radar processing [7]. Particular areas where the tools may prove useful in the future are the growing use of kernel-based methods, and low-rank approximations in machine learning and in bioinformatics. Kernel learning [33], kernel completion [30], and the use of Bregman divergence to address matrix nearness problems $[11,19]$ (the natural distance on the cone is a Bregman divergence) are exemplary illustrations of areas that could benefit from the computational tools introduced in this paper. 


\section{Appendix}

\subsection{Reductive homogeneous space and invariant met- rics}

Let us choose the identification $P_{n} \cong \mathrm{Gl}(\mathrm{n}) / \mathrm{O}(\mathrm{n})$ as a starting point. Every matrix of the Lie algebra $\mathfrak{g l}(n)=T_{I} \mathrm{Gl}(\mathrm{n})=\mathbb{R}^{n \times n}$ can be expressed as the sum of its symmetric part and its skew-symmetric part. Thus $\mathfrak{g l}(n)=$ $\mathfrak{m}+\mathfrak{s o}(n)$ is the direct sum of the symmetric matrices $\mathfrak{m}$ and the Lie algebra $\mathfrak{s o}(n)=T_{I} \mathrm{O}(\mathrm{n})$, made of skew-symmetric matrices. Moreover, for any $S \in \mathfrak{m}$ and $O \in \mathrm{O}(\mathrm{n})$ we have $A d_{O}(S)=O S O^{-1}=O S O^{T} \in \mathfrak{m}$. Thus $A d_{\mathrm{O}(\mathrm{n})}(\mathfrak{m}) \subset \mathfrak{m}$. The existence of $\mathfrak{m}$ such that $\mathfrak{g l}(n)=\mathfrak{m}+\mathfrak{s} \mathfrak{o}(n)$ is a direct sum, and $A d_{\mathrm{O}(\mathrm{n})}(\mathfrak{m}) \subset \mathfrak{m}$ proves that $\mathrm{Gl}(\mathrm{n}) / \mathrm{O}(\mathrm{n})$ can be called a reductive homogeneous space.

We provide some more information for the readers who are familiar with standard ideas of Lie group theory. Let $\{Z(t), t>0\}$ be a trajectory in Gl(n). $\left\{Z_{1}(t)=Z(t) O(t), t>0\right\}$ with $O(t) \in \mathrm{O}(\mathrm{n})$ represents the same trajectory in $P_{n}$. Let $\omega(t)=Z^{-1} \frac{d}{d t} Z \in \mathfrak{g l}(n)$ and $\omega_{1}(t)=Z_{1}^{-1} \frac{d}{d t} Z_{1}$. We have $\omega(t)=A d_{O(t)}\left(\omega_{1}(t)-O(t)^{-1} \frac{d}{d t} O(t)\right)$ (see [18]). Decomposing $\omega, \omega_{1}$ in their symmetric and skew-symmetric parts, we see that any $A d_{\mathrm{O}(\mathrm{n})}$-invariant scalar product on $\mathfrak{m}$ will allow the construction of a well-defined GL(n)-invariant metric on the homogeneous space $\mathrm{Gl}(\mathrm{n}) / \mathrm{O}(\mathrm{n})$ corresponding to this scalar product at $I$. All $A d_{\mathrm{O}(\mathrm{n})}$-invariant scalar products on the symmetric matrices are given up to a constant scale factor by $\operatorname{Tr}\left(X_{1}^{T} X_{2}\right)+\beta \operatorname{Tr}\left(X_{1}\right) \operatorname{Tr}\left(X_{2}\right)$ with $\beta>-\frac{1}{n}$. Indeed, they are derived from rotationally invariant norms $\|X\|^{2}$ on symmetric matrices. Thus they can only depend on the scalar invariants $\operatorname{Tr}(X), \operatorname{Tr}\left(X^{2}\right), \operatorname{Tr}\left(X^{3}\right)$, etc. As they are quadratic functions they can only depend on $\operatorname{Tr}(X)^{2}$ and $\operatorname{Tr}\left(X^{2}\right)$. The condition on $\beta$ ensures positive definiteness (see, e.g., [27]).

\subsection{Several proofs}

Proof of Theorem 2 Let $U(t)$ be a geodesic linking $U_{A}$ and $U_{B}$ in $\mathrm{V}_{\mathrm{n}, \mathrm{p}}$ and $R^{2}(t)$ a geodesic linking $R_{A}^{2}$ and $R_{B}^{2}$ in $\mathrm{P}_{\mathrm{p}}$, where $\left(U_{A}, R_{A}^{2}\right)$ and $\left(U_{B}, R_{B}^{2}\right)$ are representatives of $A$ and $B$ defined via SVD (11). By corollary 3.57 of [24] $\left(U(t), R^{2}(t)\right)$ is a geodesic curve in the structure space $\mathrm{V}_{\mathrm{n}, \mathrm{p}} \times \mathrm{P}_{\mathrm{p}}$ endowed with the metric $g^{S P}$. The choice of the representatives is such that for every $t>0, \frac{d}{d t} U(t)$ is orthogonal to $U(t)$, as it is an element of the horizontal space of $\operatorname{Gr}(\mathrm{p}, \mathrm{n})=\mathrm{V}_{\mathrm{n}, \mathrm{p}} / \mathrm{O}(\mathrm{p})$. This has two consequences. First the length of the 
geodesic in the structure space is

$$
d^{2}(A, B)=d_{\mathbb{G} r(p, n)}^{2}(\operatorname{range}(A), \operatorname{range}(B))+k d_{\mathrm{P}_{\mathrm{p}}}^{2}\left(R_{A}^{2}, R_{B}^{2}\right) .
$$

Because the Riemannian distance in $\operatorname{Gr}(\mathrm{p}, \mathrm{n})$ is $\|\Theta\|_{F}[12,1]$ and the Riemannian distance in $\mathrm{P}_{\mathrm{p}}$ is $\left\|\log R_{A}^{-1} R_{B}^{2} R_{A}^{-1}\right\|_{F}$, we obtain

$$
d^{2}(A, B)=\|\Theta\|_{F}^{2}+k\left\|\log R_{A}^{-1} R_{B}^{2} R_{A}^{-1}\right\|_{F}^{2}
$$

Then $\frac{d}{d t}\left(U(t), R^{2}(t)\right)$ belongs to the horizontal space $\mathcal{H}_{\left(U(t), R^{2}(t)\right)}$ for all $t>0$, that is, $\left(U(t), R^{2}(t)\right)$ is a horizontal curve. As $g^{S P}$ induces the metric (10) on the quotient manifold, the length $l\left(\gamma_{A \rightarrow B}\right)$ of $\gamma_{A \rightarrow B}$ in the quotient space $\left(\mathrm{V}_{\mathrm{n}, \mathrm{p}} \times \mathrm{P}_{\mathrm{p}}\right) / \mathrm{O}(\mathrm{p})$ is also $d(A, B)$, proving $(15)$.

Uniqueness. First of all suppose the $\sigma_{i}$ 's are distinct. According to the uniqueness of the SVD in (11), the matrices $U_{A}$ and $U_{B}$ are unique (up to a joint multiplication of any columns by -1 , which is an orthogonal transformation) and do not depend on the choice of $V_{A}$ and $V_{B}$. Under the less restrictive assumption $\sigma_{p-1}>0$, suppose there exists $1 \leq i \leq p-1$ such that $\sigma_{i}=\sigma_{i+1}$. Then $\sigma_{i}>0$ and the SVD yields $u_{i}^{A}, u_{i}^{B}, u_{i+1}^{A}, u_{i+1}^{B}$ such that $\left(u_{i}^{A}\right)^{T} u_{i}^{B}=\left(u_{i+1}^{A}\right)^{T} u_{i+1}^{B}=\sigma_{i}$. For the sake of simplicity we assume there are only 2 principal vectors associated to $\sigma_{i}$. The generalization to an arbitrary number of vectors is straightforward and leads to the same conclusion. The SVD yields non-unique principal vectors, since any normalized linear combination of $u_{i}$ and $u_{i+1}$ is still a principal vector. Let $u^{A}=a u_{i}^{A}+b u_{i+1}^{A}$ and $u^{B}=c u_{i}^{B}+d u_{i+1}^{B}$ be other principal vectors associated to $\sigma_{i}$. By definition $\left(u^{A}\right)^{T} u^{B}=\sigma_{i}$ and $\left(u^{A}\right)^{T} u^{A}=\left(u^{B}\right)^{T} u^{B}=1$. Since $\left(u_{i}^{A}\right)^{T} u_{i+1}^{B}=0$ we have $(a c+b d) \sigma_{i}=\sigma_{i}$. It implies $a=c$ and $b=d$ (Cauchy-Schwartz equality) and, necessarily, $u^{A}$ and $u^{B}$ are obtained from $u_{i}^{A}, u_{i+1}^{A}$, and $u_{i}^{B}, u_{i+1}^{B}$ via the same orthogonal transformation (unless $\sigma_{i}=0$, which is impossible). We proved the principal vectors $U_{A}$ and $U_{B}$ are defined up to a joint multiplication on the right by a block orthogonal matrix $P \in \mathrm{O}(\mathrm{p})$, each block being associated to the same eigenvalue. Let $U_{A} P=U_{A}^{\prime}$ and $U_{B} P=U_{B}^{\prime}$, and let $A=U_{A}^{\prime} R_{A}^{\prime 2}\left(U_{A}^{\prime}\right)^{T}$ and $B=U_{B}^{\prime} R_{B}^{\prime 2}\left(U_{B}\right)^{\prime T}$. We have $R_{A}^{\prime 2}=P^{T} R_{A}^{2} P, R_{B}^{\prime 2}=P^{T} R_{B}^{2} P$ and $l\left(\gamma_{A \rightarrow B}\right)$ is unchanged since $d_{\mathrm{P}_{\mathrm{p}}}\left(R_{A}^{2}, R_{B}^{2}\right)=d_{\mathrm{P}_{\mathrm{p}}}\left(R_{A}^{\prime 2}, R_{B}^{\prime 2}\right)$. Note that the reciprocal of this result is also true.

Invariances. Using the preceding paragraph, we know that (15) is well defined under the basic assumption $\sigma_{p-1}>0 \Leftrightarrow \theta_{p-1}<\pi / 2$. Thus the following features are sufficient to complete the proof:

- For $\mu \in \mathbb{R}$ we have $\mu^{2} A=U_{A}\left(\mu^{2} R_{A}^{2}\right) U_{A}^{T}$. Since we have $d_{\mathrm{P}_{\mathrm{p}}}\left(\mu^{2} R_{A}^{2}, \mu^{2} R_{B}^{2}\right)=$ $d_{\mathrm{P}_{\mathrm{p}}}\left(R_{A}^{2}, R_{B}^{2}\right)$ we have $l\left(\gamma_{\mu^{2} A \rightarrow \mu^{2} B}\right)=l\left(\gamma_{A \rightarrow B}\right)$. 
- Let $O \in \mathrm{O}(\mathrm{n})$, and let $U_{A}$ and $U_{B}$ be the principal vectors associated to $A, B$. Then $U_{A}^{\prime}=O U_{A}$ and $U_{B}^{\prime}=O U_{B}$ are principal vectors associated to $O A O^{T}$ and $O B O^{T}$ since orthogonal transformations preserve the angles: $\left(O U_{A}\right)^{T} O U_{B}=U_{A}^{T} U_{B}$, so the Grassman distance is unchanged. Since $O A O^{T}=U_{A}^{\prime} R_{A}^{2} U^{\prime T}$ and $O B O^{T}=U_{B}^{\prime} R_{B}^{2} U_{B}^{\prime T}$ we see that $R_{A}^{2}, R_{B}^{2}$ are also unchanged by the transformation and $l\left(\gamma_{O A O^{T} \rightarrow O B O^{T}}\right)=l\left(\gamma_{A \rightarrow B}\right)$.

- $A^{\dagger}=U_{A} R_{A}^{-2} U_{A}^{T}$ is the pseudoinverse of $A$. $\quad l\left(\gamma_{A \rightarrow B}\right)-l\left(\gamma_{A^{\dagger} \rightarrow B^{\dagger}}\right)=$ $d_{\mathrm{P}_{\mathrm{p}}}\left(R_{A}^{-2}, R_{B}^{-2}\right)-d_{\mathrm{P}_{\mathrm{p}}}\left(R_{A}^{2}, R_{B}^{2}\right)=0$.

Proof of Proposition 2 First, as $\gamma_{A \rightarrow B}$ induces a non-minimal path connecting $A$ and $B$ in $\mathrm{S}^{+}(\mathrm{p}, \mathrm{n})$ we have $l^{2}\left(\gamma_{A \rightarrow B}\right) \geq d_{\mathrm{S}^{+}(\mathrm{p}, \mathrm{n})}(A, B)$. Then, let $\gamma(t)$ be a geodesic linking $A$ and $B$ in $\mathrm{S}^{+}(\mathrm{p}, \mathrm{n})$, with $\gamma(0)=A, \gamma(1)=B$ and $d_{\mathrm{S}^{+}(\mathrm{p}, \mathrm{n})}(A, B)$ its length. As proved in the sequel $\gamma(t)$ can be globally horizontally lifted in the structure space $\mathrm{V}_{\mathrm{n}, \mathrm{p}} \times \mathrm{P}_{\mathrm{p}}$. The horizontal lift $\tilde{\gamma}(t)$ at $\left(U_{A}, R_{A}\right)$ is a horizontal curve in the structure space $\mathrm{V}_{\mathrm{n}, \mathrm{p}} \times \mathrm{P}_{\mathrm{p}}$ connecting the two fibers. Therefore there exists $O \in O(p)$ such that $\tilde{\gamma}(1)=$ $\left(U_{B} O, O^{T} R_{B}^{2} O\right)$, where $\left(U_{B}, R_{B}^{2}\right)$ is defined via the SVD (11). The length of $\tilde{\gamma}$ in the structure space is also $d_{\mathrm{S}^{+}(\mathrm{p}, \mathrm{n})}(A, B)$. Since $\tilde{\gamma}$ is not necessarily a minimal curve in the structure space we have $d_{\mathrm{S}^{+}(\mathrm{p}, \mathrm{n})}^{2}(A, B) \geq d_{\mathrm{V}_{\mathrm{n}, \mathrm{p}}}^{2}\left(U_{A}, U_{B} O\right)+$ $k d_{\mathrm{P}_{\mathrm{p}}}^{2}\left(R_{A}^{2}, O^{T} R_{B}^{2} O\right)$. Because distances in $\operatorname{Gr}(p, n)$ are shorter than those in $\mathrm{V}_{\mathrm{n}, \mathrm{p}}$, this implies $d_{\mathrm{S}^{+}(\mathrm{p}, \mathrm{n})}^{2}(A, B) \geq d_{\mathrm{Gr}(p, n)}^{2}\left(\operatorname{range}\left(U_{A}\right)\right.$, range $\left.\left(U_{B} O\right)\right)+k d_{\mathrm{P}_{\mathrm{p}}}^{2}\left(R_{A}^{2}, O^{T} R_{B}^{2} O\right)$. Finally we proved

$$
\begin{aligned}
l^{2}\left(\gamma_{A \rightarrow B}\right) & \leq d_{\operatorname{Gr}(p, n)}^{2}\left(\operatorname{range}\left(U_{A}\right), \operatorname{range}\left(U_{B}\right)\right)+k d_{\mathrm{P}_{\mathrm{p}}}^{2}\left(R_{A}^{2}, O^{T} R_{B}^{2} O\right)+k d_{\mathrm{P}_{\mathrm{p}}}^{2}\left(O^{T} R_{B}^{2} O, R_{B}^{2}\right) \\
& \leq d_{\mathrm{S}^{+}(\mathrm{p}, \mathrm{n})}^{2}(A, B)+k d_{\mathrm{P}_{\mathrm{p}}}^{2}\left(R_{B}^{2}, O^{T} R_{B}^{2} O\right)
\end{aligned}
$$

Proof of Proposition 3 The structure space is geodesically complete (it is a product of two geodesically complete Riemannian manifolds whose geodesics are product of geodesics). Thus horizontal geodesics are defined for all $t \in \mathbb{R}$.

A manifold is geodesically complete if and only if it is complete as a metric space (see Theorem 10.3 Ch I in [16]). Thus the structure space is complete. To prove that the quotient space $\mathrm{S}^{+}(\mathrm{p}, \mathrm{n})$ is geodesically complete it suffices to prove that it is complete. Let $\left\{u_{m}\right\}$ be a Cauchy sequence in the quotient space $\mathrm{S}^{+}(\mathrm{p}, \mathrm{n})$. One can find a broken geodesic $\gamma(t)$ linking the $u_{i}$ 's such that $\gamma\left(t_{i}\right)=u_{i}$. Consider an horizontal lift $\tilde{\gamma}$ of $\gamma$ (it is well defined as proved below). The distance between $\tilde{\gamma}\left(t_{i}\right)$ and $\tilde{\gamma}\left(t_{j}\right)$ in the structure space is $d_{\mathrm{S}^{+}(\mathrm{p}, \mathrm{n})}\left(\gamma\left(t_{i}\right), \gamma\left(t_{j}\right)\right)=d_{\mathrm{S}^{+}(\mathrm{p}, \mathrm{n})}\left(u_{i}, u_{j}\right)$ and thus $\left\{\tilde{\gamma}\left(t_{m}\right)\right\}$ is a cauchy sequence 
in the structure space. Thus it converges to a point $\tilde{p}=\left(U, R^{2}\right)$. Let us prove $\left\{u_{m}\right\}$ converges to $p=U R^{2} U^{T}$. Thanks to the exponential map in the structure space, $\tilde{\gamma}\left(t_{i}\right)$ can be identified to an element $\xi_{i}=\left(\Delta_{i}+U \Omega_{i}, D_{i}\right) \in$ $T_{\left(U, R^{2}\right)} \mathrm{V}_{\mathrm{n}, \mathrm{p}} \times \mathrm{P}_{\mathrm{p}}$ for $i$ large enough. The geodesic distance in the structure space between $\tilde{\gamma}\left(t_{i}\right)$ and $\tilde{p}$ is $g^{S P}\left(\xi_{i}, \xi_{i}\right)=\left\|\Omega_{i}\right\|^{2}+\left\|\Delta_{i}\right\|^{2}+k \operatorname{Tr}\left(\left(D_{i} R^{-2}\right)^{2}\right)$ and thus $\left\|\Omega_{i}\right\| \rightarrow 0$. Thus the coordinate of $\tilde{\gamma}\left(t_{i}\right)$ along the fiber tends to zero so $d_{\mathrm{S}^{+}(\mathrm{p}, \mathrm{n})}\left(U_{i} R_{i}^{2} U_{i}^{T}, U R^{2} U^{T}\right) \rightarrow 0$.

Any geodesic $\gamma$ of $\mathrm{S}^{+}(\mathrm{p}, \mathrm{n})$ can be globally horizontally lifted. This result was shown by R. Hermann when the horizontal and vertical spaces are orthogonal. The result holds in our case and the proof is unchanged. Let us recall the main points of the proof : let $\tilde{\gamma}_{p}$ is the maximal horizontal lift of $\gamma$ at $p$, defined on, say $\left[0, \epsilon\left[\right.\right.$. It is enough to show that $\tilde{\gamma}_{p}$ can be extended at $\epsilon$. The continuity of $\gamma$ and the completeness of the structure space imply the existence of a limit point $q=\lim _{t \rightarrow \epsilon} \tilde{\gamma}_{p}(t)$.

\section{Acknowledgment}

The authors wish to thank P.-A. Absil for several discussions on the paper.

\section{References}

[1] P.-A. Absil, R. Mahony, And R. Sepulchre, Riemannian geometry of Grassmann manifolds with a view on algorithmic computation, Acta Appl. Math., 80 (2004), pp. 199-220.

[2] P.-A. Absil, R. Mahony, And R. Sepulchre, Optimization Algorithms on Matrix Manifolds, Princeton University Press, Princeton, NJ, 2007.

[3] P.-A. Absil, M. Ishteva, L. De Lathauwer, and S. Van HufFEL, A geometric Newton method for Oja's vector field, Neural Comput., 21 (2009), pp. 1415-1433.

[4] T. Ando, C.-K. Li, And R. Mathias, Geometric means, Linear Algebra Appl., 385 (2004), pp. 305-334.

[5] T. Ando, Topics on Operator Inequalities, Division of Applied Mathematics, Hokkaido University, Sapporo, Japan, 1978.

[6] V. Arsigny, P. Fillard, X. Pennec, and N. Ayache, Geometric means in a novel vector space structure on symmetric positive-definite matrices, SIAM J. Matrix Anal. Appl., 29 (2007), pp. 328-347. 
[7] F. BARBAREsco, Innovative tools for radar signal processing based on Cartan's geometry of symmetric positive-definite matrices and information geometry, in Proceedings of the IEEE International Radar Conference, Rome, Italy, 2008.

[8] S. Bonnabel, Ph. Martin, and P. Rouchon, Symmetry-preserving observers, IEEE Trans. Automat. Control, 53 (2008), pp. 2514-2526.

[9] S. Boyd and L. Vandenberghe, Convex Optimization, Cambridge University Press, Cambridge, UK, 2004.

[10] J. Burbea AND C. R. RAO, Entropy differential metric, distance and divergence measures in probability spaces: A unified approach, J. Multivariate Anal., 12 (1982), pp. 575-596.

[11] I. S. Dhillon And J. A. Tropp, Matrix nearness problems with Bregman divergences, SIAM J. Matrix Anal. Appl., 29 (2007), pp. 1120-1146.

[12] A. Edelman, T. A. Arias, And S. T. Smith, The geometry of algorithms with orthogonality constraints, SIAM J. Matrix Anal. Appl., 20 (1998), pp. 303-353.

[13] J. Faraut and A. Koranyi, Analysis on Symmetric Cones, Oxford University Press, New York, 1994.

[14] P. D. Fletcher And S. Joshi, Riemannian geometry for the statistical analysis of diffusion tensor data, Signal Processing, 87 (2007), pp. 250-262.

[15] G. H. Golub And C. F. Van Loan, Matrix Computations, John Hopkins University Press, Baltimore, MD, 1983.

[16] S. Hegalson, Differential Geometry and Symmetric Spaces, Pure Appl. Math., Academic Press, New York and London, 1962.

[17] J. Jost, Riemannian Geometry and Geometric Analysis, SpringerVerlag, Berlin, 1995.

[18] F. KLeIn, Development of Mathematics in the 19th Century, Math Sci Press, Brookline, MA, 1979.

[19] B. Kulis, M. Sustik, And I. S. Dhillon, Learning low-rank kernel matrices, in Proceedings of the 23rd International Conference on Machine Learning, Pittsburgh, PA, 2006, ACM, New York, 2006, pp. $505-512$. 
[20] M. Lovrić, M. Min-Oo, AND E. A. RuH, Multivariate normal distributions parametrized as a Riemannian symmetric space, J. Multivariate Anal., 74 (2000), pp. 36-48.

[21] M. MonkHer, A differential geometric approach to the geometric mean of symmetric positive-definite matrices, SIAM J. Matrix Anal. Appl., 26 (2005), pp. 735-747.

[22] Yu. E. Nesterov and M. J. Todd, On the Riemannian geometry defined for self-concordant barriers and interior point methods, Found. Comput. Math., 2 (2002), pp. 333-361.

[23] P. J. Olver, Equivalence, Invariants, and Symmetry, Cambridge University Press, Cambridge, UK, 1995.

[24] B. O'Neill, Semi-Riemannian Geometry, Pure Appl. Math. 103, Academic Press, New York, 1983.

[25] X. Pennec, Intrinsic statistics on Riemannian manifolds: Basic tools for geometric measurements, J. Math. Imaging Vision, 25 (2006), pp. $127-154$

[26] X. Pennec, P. Fillard, and N. Ayache, A Riemannian framework for tensor computing, International Journal of Computer Vision, 66 (2006), pp. 41-66.

[27] X. Pennec, Habilitation a diriger les recherches, Universite de Nice, Nice, France, 2006.

[28] D. Petz and R. Temesi, Means of positive numbers and matrices, SIAM J. Matrix Anal. Appl., 27 (2005), pp. 712-720.

[29] W. Pusz And S. L. Woronowicz, Functional calculus for sesquilinear forms and the purification map, Rep. Mathematical Phys., 8 (1975), pp. 159-170.

[30] B. Scholkopf, K. Tsuda, And J.-Ph. Vert, Kernel Methods in Computational Biology, MIT Press, Cambridge, MA, 2004.

[31] L. T. SkovgaARd, A Riemannian geometry of the multivariate normal model, Scand. J. Statist., 11 (1984), pp. 211-223.

[32] S. T. Smith, Covariance, subspace, and intrinsic Cramér-Rao bounds, IEEE Trans. Signal Process., 53 (2005), pp. 1610-1630. 
[33] K. Tsuda, G. Ratsch, and M. K. Warmuth, Matrix exponentiated gradient updates for on-line learning and Bregman projection, J. Mach. Learn. Res., 36 (2005), pp. 995-1018.

[34] M. Zerai and M. Moakher, The Riemannian geometry of the space of positive-definite matrices and its application to the regularization of diffusion tensor MRI data, J. Math. Imaging Vision, submitted. 